working conditions and employment conditions on prospective return to work and work ability following injury will be made.

\section{RETURN TO WORK AND WORK ABILITY AFTER INJURY: RESULTS FROM THE PROSPECTIVE OUTCOME OF INJURY STUDY}

R Lilley*, S Derrett, S Ameratunga, G Davie Correspondence: Injury Prevention Research Unit, PO Box 913 University of Otago, Dunedin 9054, New Zealand

10.1136/ip.2010.029215.270

Background Return to work and work ability are considered major markers of rehabilitation following injury. Prolonged work absence and work disability result in significant personal and social costs, therefore improvements in return to work and work ability will have benefits for injured workers and society. The Prospective Outcomes of Injury Study (POIS) is a cohort study following the outcomes of injured New Zealanders.

Aim This work-related component of the study aims to determine preinjury predictors of return to work and work ability for 2850 New Zealanders recruited into the POIS cohort.

Methods The 2850 participants for this study comprise a sample of individuals aged 18-65 years referred to the national injury compensation scheme (ACC) for an entitlement claim (ie, injury severe enough to warrant 1 week off work or home/ transportation assistance) during the period December 2007 to August 2009. Quantitative data are collected by questionnaire at 3, 5, 12 \& 24 months after injury.

Results and Conclusion This presentation will discuss results from 3 and 5 month interviews describing the injury outcomes relevant to return to work and return to work ability in the cohort. Of the 2850 participants, 2618 were workforce active. Presentation of analyses examining the impact of preinjury work factors, including psychosocial and physical 